\title{
ALÉM DO PLANO DE ENSINO: ATIVIDADES EXTRACURRICULARES E SUA IMPORTÂNCIA
}

Amanda de Carvalho - amanndaat@gmail.com

Universidade Federal de Santa Catarina - UFSC

Rua Dona Francisca, 8300

89219-600 - Joinville - Santa Catarina

Heloisa Prestes da Silva - heloisaprestes24@gmail.com

Universidade Federal de Santa Catarina - UFSC

Rua Dona Francisca, 8300

89219-600 - Joinville - Santa Catarina

Carlos Mauricio Sacchelli - carlos.sacchelli@gmail.com

Universidade Federal de Santa Catarina - UFSC

Rua Dona Francisca, 8300

89219-600 - Joinville - Santa Catarina

Resumo: A busca pelo conhecimento é um instinto intrínseco humano e isso se dá muito além de iniciar uma graduação ou um novo idioma. Diversas são as habilidades e competências de um profissional necessárias atualmente, desta maneira, as atividades extracurriculares vêm se tornando uma realidade cada vez mais presente em alunos de instituições de ensino superior. Assim, o objetivo principal deste artigo é de ressaltar a importância na participação de atividades extracurriculares para que o estudante tenha um grande desenvolvimento pessoal e profissional. Para este estudo, foi realizado uma pesquisa com estudantes participantes dos cursos de AutoCAD e Civil 3D, ministrados pelos membros do Programa de Educação Tutorial na Universidade Federal de Santa Catarina, campus Joinville. Pode-se concluir que atividades extracurriculares na graduação contribuem para o crescimento pessoal e profissional dos estudantes.

Palavras-chave: Atividades extracurriculares. Cursos. Ensino.

\section{INTRODUÇÃO}

O ingresso em meios acadêmicos é uma divisa entre a vida adulta em grande parte da comunidade universitária. Essa transição traz diversas mudanças tanto na rotina quanto no comportamento dos estudantes, tais como o desenvolvimento nas áreas cognitivas, social e afetiva, ganhos em habilidades intelectuais, desenvolvimento de conhecimentos específicos e domínio em dimensões atitudinais, psicossociais e morais, e junto com essas mudanças vem o exercício da autonomia (ASTIN, 1993; KUH, 1995; PASCARELLA, TERENZINI, 1991, 2005).

A busca por atividades que vão além das descritas no Projeto Pedagógico de Curso exercitam a autonomia universitária, além de trazer um diferencial para a formação do futuro 
profissional. (ASTIN, 1993). Além disso, essas atividades podem minimizar os índices de retenção e evasão pois priorizam não só as notas, mas o desenvolvimento de novos conhecimentos, ampliando a qualidade da educação e favorecendo a aprendizagem destes estudantes. (NODARI, 2018).

Capovilla e Santos (2001) mostraram isso de forma experimental, aplicando uma pesquisa com estudantes do curso de Odontologia. Os estudantes participavam de atividades extracurriculares e as alterações percebidas pelos participantes foram mudança no raciocínio reflexivo, melhora e desenvolvimento de habilidades acadêmicas, competência social e autonomia. Com este estudo, os dados coletados mostram a importância das atividades extracurriculares e salientam o benefício no desenvolvimento do estudante de forma geral (BAXTER-MAGOLDA, 1992; PASCARELLA e TERENZINI, 1991).

O envolvimento de estudantes em atividades extracurriculares é um tema de grande destaque em pesquisas brasileiras, que vêm sendo desenvolvidas desde a década de 90 . Destes estudos, alguns dados coletados permitiram perceber que a motivação e autonomia para o ingresso em atividades extracurriculares podem ser correlacionadas com a qualidade de identidade profissional percebida pelo estudante e seu comprometimento com a universidade e com a sua formação (Bardagi, Hutz, 2012).

Fior e Mercuri (2009) pontuam que o caminho efetivo para uma boa aprendizagem se baseia na criação de condições motivadoras e inspiradoras, que atraiam os estudantes a investirem seu tempo e energia em atividades extracurriculares. Em um estudo realizado por Oliveira, Santos e Dias (2016), os autores verificaram que os maiores motivos pelo qual os estudantes buscam as atividades extracurriculares se dá com a necessidade da responsabilidade com o desenvolvimento profissional e o fato de que os conhecimentos adquiridos através dos professores em sala de aula já não são mais suficientes para serem um profissional de excelência.

Além dos benefícios pessoais e internos que a participação em atividades extracurriculares traz para os estudantes universitários, Fior e Mercuri (2009) dizem que ainda faz com que os estudantes adquiram identificação com o curso, experimentando atividades práticas e teóricas diretamente ligadas com o exercício da profissão em questão.

Por mais que os estudos trazem estatísticas sobre a real importância das atividades extracurriculares na vida acadêmica, a opinião dos estudantes vai contra a estatística. Essa divergência se vê grandemente com a separação de dois grandes grupos de estudantes, calouros e formandos. Calouros (estudantes da primeira metade de fases do curso) tendem a não enxergar com clareza a importância das atividades extracurriculares, já os formandos (estudantes da segunda metade de fases do curso) tendem a exaltar a importância delas, percebendo que o pensamento pode ser mudado conforme o amadurecimento do universitário dentro do âmbito acadêmico (OLIVEIRA, SANTOS, DIAS, 2016).

Supõem-se que ao participar de atividades extracurriculares, o estudante desenvolva o sentimento de pertencimento, ao desenvolver um projeto ou habilidade, além de trazer sentimentos de satisfação com a conclusão de uma tarefa e auxilia no desempenho acadêmico, já que os sentimentos de satisfação os impulsiona a buscarem mais disso, tornando um sistema retroalimentado.

Dentre as modalidades de atividades extracurriculares, podemos citar algumas como monitorias, projetos de pesquisa e extensão, grupos de estudos, participação em órgãos estudantis, congressos e eventos científicos, estágios entre outros (Bardagi, Hutz, 2012; Fior, Mercuri, 2009).

As novas Diretrizes Curriculares Nacionais para os cursos de graduação em Engenharia, publicadas em abril de 2019 pelo Ministério da Educação (MEC), tem como ideia principal que as instituições de ensino superior formem profissionais mais completos e que essa 
formação seja baseada por competências, foco na prática, aprendizagem ativa e uma maior flexibilidade na constituição do currículo. Algumas dessas competências podem ser alcançadas com a formação extracurricular dos estudantes (RECKZIEGEL, 2019).

O Programa de Educação Tutorial (PET), criado pela Coordenadoria de Apoio ao Pessoal de Ensino Superior, tem como objetivo promover aos estudantes a oportunidade de ensino, pesquisa e extensão. Orientados por um tutor, realizam atividades extracurriculares que complementam a formação acadêmica e atendem às necessidades do próprio curso de graduação (BRASIL, 2018).

Vários PETs desenvolvem atividades extracurriculares para estudantes dos cursos, como por exemplo o PET de Engenharia Elétrica da Universidade Federal de Juiz de Fora realiza semanalmente, o projeto denominado LinusBot, que envolve a montagem e programação de um robô pelos jovens calouros, que têm a chance de aplicar um conhecimento teórico na prática, o que torna o início da graduação mais motivador. (VALLE et al.; 2018).

Para o caso que será apresentado neste trabalho, será estudada a aplicação do curso básico do software AutoDesk AutoCAD e AutoDesk Civil 3D para estudantes de graduação da Universidade Federal de Santa Catarina, no Campus de Joinville, onde há 7 (sete) graduações de engenharia diferentes e 1 (um) curso de Ciência e Tecnologia. Para este curso não foram colocadas restrições quanto ao curso de graduação feito pelo aluno e tampouco quanto ao número mínimo de períodos cursados.

Segundo a própria empresa, o AutoCAD é um software de CAD (projeto auxiliado por computador —do inglês Computer-Aided Design) para o desenvolvimento de projetos em 2D e 3D precisos por arquitetos, engenheiros e profissionais da construção civil. A Figura 1 mostra um exemplo de projeto executado em AutoCAD.

Figura 1 - Projeto AutoCAD
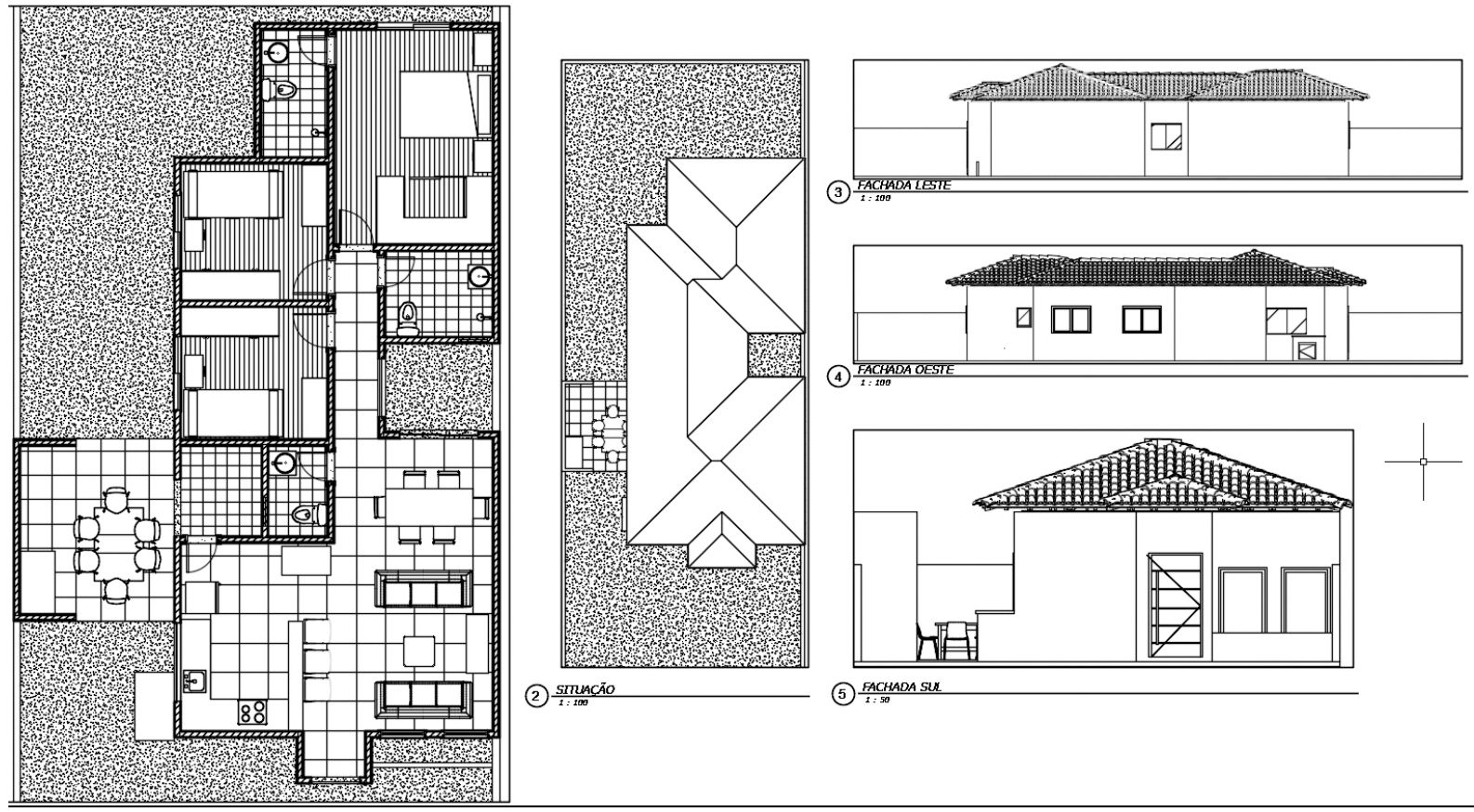

(5) $\frac{\text { FACHADA SUR }}{1: 50}$

Fonte: SAVOY (2016) 
Já o software AutoDesk Civil 3D é um programa de apoio a plataforma BIM (Building Information Modeling), com a integração de recursos de aprimoramento de desenhos, projetos e construção. Dá suporte para uma variedade de projetos de infraestrutura civil que incluem ferrovias, estradas e rodovias, desenvolvimento de terrenos, aeroportos, drenagem, águas pluviais e esgoto e estruturas civis. A Figura 2 mostra um exemplo de projeto executado em AutoDesk Civil 3D.

Figura 2 - Projeto em Civil 3D

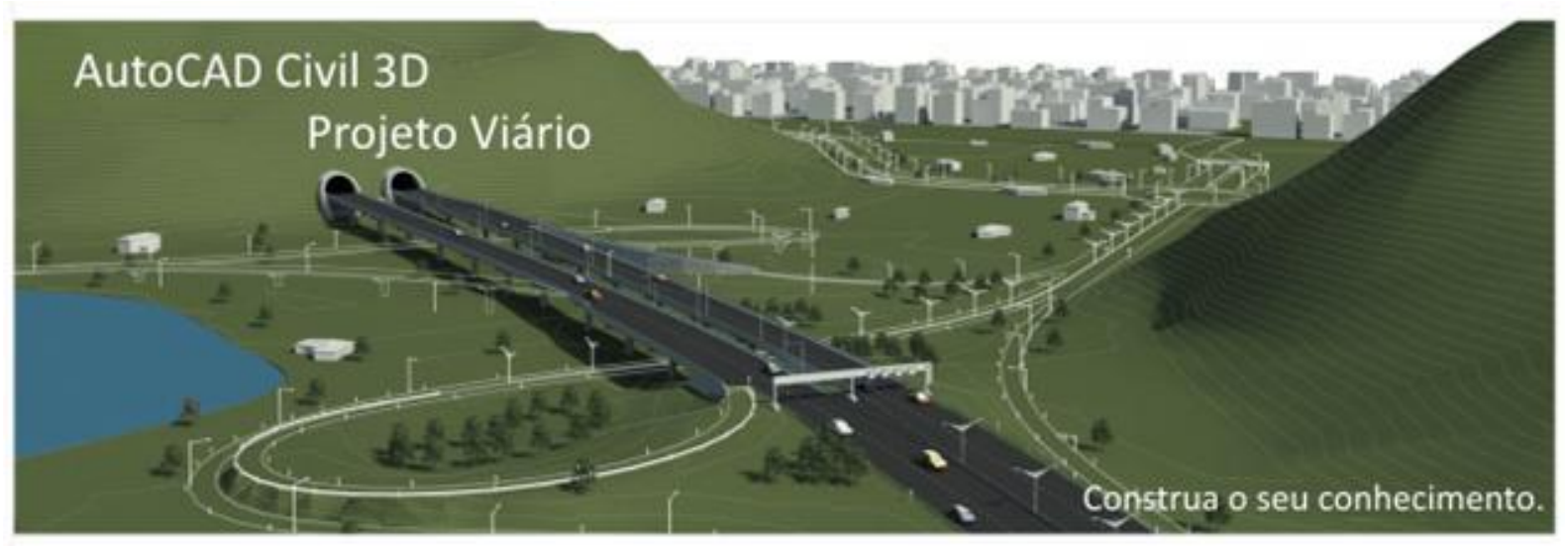

Fonte: VIEIRA (2010)

\section{DESENVOLVIMENTO DA ATIVIDADE}

Ambos os cursos foram aplicados nas dependências da Universidade Federal de Santa Catarina, no campus Joinville. Cada curso teve a duração de oito horas, ministrados duas vezes na semana, tendo duas horas de duração.

Os cursos contaram com o auxílio do software e completamente desenvolvido nele. Os conteúdos propostos foram de cunho básico. O curso de AutoCAD abordou os temas de introdução ao software e comandos básicos, layers e estudo de espessura de linha, configuração de texto e escala de portview e plotagem e impressão. Já o curso de Civil 3D contou com os temas de introdução ao software, configuração e interpretação de arquivo de pontos, superfícies, alinhamentos, perfil topográfico e projetado, cálculo de volume de corte e aterro e plotagem.

Os participantes inscritos nos cursos receberam as instruções de utilização dos softwares e de desenvolvimento dos projetos do ministrante, contando com o auxílio de um monitor para dúvidas. Além dos conteúdos teóricos ministrados em sala, juntamente com o material impresso, os participantes contaram com a execução de projetos passo-a-passo em tempo real e o desenvolvimento de exercícios para a prática do conteúdo aprendido em sala.

Para fins de avaliação da atividade, algumas perguntas foram selecionadas e feitas aos participantes do curso, a fim de saber o nível de conhecimento absorvido em sala, a eficácia dos métodos aplicados, satisfação com o aprendizado, além do tema principal, a importância do aprendizado com atividades extracurriculares para a formação acadêmica. 
"Os desafios para formar hoje o engenheiro do amanhã"

Foi enviado aos 11 participantes dos cursos um formulário eletrônico com as seguintes perguntas:

1. Qual graduação faz na UFSC?

2. Qual era o nível de conhecimento sobre o software antes da participação no(s) curso(s)?

3. Se você pudesse nivelar os conhecimentos adquiridos no(s) curso(s) realizado(s), qual seria?

4. Você acha que os métodos utilizados para o ensino do software (instruções projetadas passo-a-passo, exercícios desenvolvidos em sala de aula e material de apoio impresso) foram eficazes para o aprendizado?

5. Qual método de ensino utilizado mais ajudou no entendimento do software durante as aulas?

6. Após a conclusão do curso, você acha que sabe o básico sobre o software?

7. Você acha que cursos extracurriculares são importantes para o desenvolvimento acadêmico?

8. Como você acha que esse(s) curso(s) irão afetar positivamente ou negativamente seu desempenho acadêmico? Descreva brevemente

Com a coleta e análise dos dados recolhidos através do formulário, podemos entender a importância das atividades extracurriculares na formação profissional em universidades.

\section{RESULTADOS E DISCUSSÕES}

Após a coleta dos dados do formulário, podemos entender um pouco mais sobre como o estudante universitário enxerga a importância das atividades extracurriculares oferecidas na universidade.

Como a pesquisa científica se dá na verificação da importância de atividades acadêmicas extracurriculares, as perguntas 4 e 5 não se tornam relevantes para a pesquisa, mas foram adicionadas a fim de se ter um feedback sobre os métodos utilizados.

Sobre qual curso de graduação os participantes fazem na universidade, 66,7\% responderam cursar Engenharia Civil de Infraestrutura, 16,7\% responderam cursar Bacharelado em Ciência e Tecnologia e 16,7\% responderam cursar Engenharia Aeroespacial.

Quanto ao nível de conhecimento do(s) software(s) antes de iniciarem o curso, $50 \%$ responderam que possuíam pouco conhecimento, como os comandos básicos, 50\% responderam não possuir conhecimento nenhum sobre o software, que não sabiam como utilizá-lo e $0 \%$ responderam possuir muito conhecimento e já saber utilizar bem o software. Este dado mostra que mesmo com algum conhecimento sobre o(s) software(s), os estudantes ainda buscam aprofundar mais o conhecimento.

Ao questionar sobre quanto conhecimento foi adquirido durante o curso, na execução das aulas e exercícios, 66,7\% responderam ter adquirido pouco conhecimento, 33,3\% responderam ter adquirido muito conhecimento e $0 \%$ respondeu não ter adquirido nenhum conhecimento. Este dado pode ser comparado e analisado à pergunta anterior. Como $50 \%$ dos participantes já iniciaram o(s) curso(s) com certo conhecimento do(s) software(s), além do nível dos cursos ser básico, pode-se ter a justificativa para a absorção de pouco conhecimento de $66,7 \%$ dos participantes. Uma solução para o aumento do índice de absorção de conteúdo ministrado no(s) curso(s), é a realização dos cursos nos níveis intermediário e avançado.

O curso tinha como objetivo ensinar conhecimentos suficientes para que os participantes tivessem um nível básico dos cursos. Quando questionados os participantes, sobre após 
finalizarem o curso, poderiam dizer que sabiam o básico sobre o curso e $75 \%$ responderam que sim, $25 \%$ responderam que talvez e $0 \%$ respondeu que não. Neste caso, a aplicação de um teste final, para o reconhecimento do aprendizado do curso daria a certeza aos participantes sobre saberem o básico sobre o(s) software(s).

Com isso, ao questionar se os participantes achavam que a execução de atividades extracurriculares é importante para o desenvolvimento acadêmico, 100\% responderam que sim. Este dado, juntamente com as respostas coletadas na pergunta 2 mostram que existe sim uma parcela de estudantes dentro da universidade que veem as atividades extracurriculares como uma ferramenta importante no desenvolvimento acadêmico.

No fim do questionário, deixamos uma questão em aberto, perguntando como os participantes acham que essas atividades afetam positiva ou negativamente (dependendo da resposta da pergunta anterior) no desempenho acadêmico. As respostas a essa pergunta variaram entre a revisão e absorção de conteúdos já aprendidos em disciplinas regulares oferecidas pelo curso de graduação, rapidez no aprendizado do software, já que o aprendizado sem o auxílio do professor é mais demorado, no desenvolvimentos de projetos no meio acadêmico, além de um diferencial para o mercado de trabalho e suporte em disciplinas onde o(s) software(s) é(são) necessário(s) e que não há capacitação dos alunos por parte dos professores.

Os cursos contaram com 11 participantes ao todo, porém somente 6 participantes responderam ao questionário, podendo dificultar a acurácia dos resultados. Para que os outros cursos consigam um número maior de inscritos algumas estratégias tem sido consideradas para as próximas edições.

\section{CONCLUSÃo}

É visível que os cursos extracurriculares trazem diversos benefícios tanto no desenvolvimento acadêmico quanto na preparação profissional para o mercado de trabalho, além de desenvolvimento pessoal e melhoras psíquicas, mas ainda vemos que os estudantes universitários ainda não visualizam essas melhoras e todas as vantagens adquiridas e a porcentagem dos que as visualizam ainda são baixas.

Conhecer a mente e o perfil dos estudantes universitários, os impedimentos ao acesso às atividades extracurriculares e suas necessidades podem ser maneiras de reverter esse quadro, trazendo mais visibilidade às atividades e profissionais mais qualificados.

A existência do PET é de suma importância tanto para os petianos, que tem a oportunidade de realizar atividades que complementam sua formação, quanto para os alunos da universidade, que se beneficiam das atividades que o programa desenvolve.

Com a realização do curso, os participantes puderam adquirir conhecimentos além de graduação, tiveram um suporte nas disciplinas que utilizam os softwares, além de um diferencial no mercado de trabalho.

A baixa adesão de estudantes na participação dos cursos realizados pelo PET EMB no campus da UFSC em Joinville é um tema passível de estudos em próximas pesquisas, aprimorando não somente os métodos de realização e produção dos cursos, mas também visando o benefício dos membros do PET e da comunidade acadêmica.

\section{Agradecimentos}

Os autores agradecem o Ministério da Educação e Cultura pela oportunidade de bolsa dentro do Programa de Educação Tutorial das Engenharias da Mobilidade (PET EMB). À 
universidade pelo apoio ao programa e apoio ao nosso projeto. Ao nosso tutor pelo suporte em todo projeto, e o suporte e trabalho conjunto dos colegas de equipe.

\section{REFERENCIAS}

ASTIN, A.W. (1993). What matters in college: Four critical years revisited. São Francisco, Jossey-Bass.

BARDAGI, M. P., \& HUTZ, C. S. (2012). Rotina acadêmica e relação com colegas e professores: Impacto na evasão universitária. Psico, 43(2), 174-184. Recuperado de http://revistaseletronicas.pucrs.br/fo/ojs/index. php/revistapsico/article/view/7870

BAXTER MAGOLDA, M.B. (1992). Cocurricular influences on college student's intellectual development. Journal of College Student Development. Washington, vol. 33, pp. 203-213.

BRASIL. Ministério da Educação. Governo Federal do Brasil (org.). Apresentação - PET. 2018. Disponível em: http://portal.mec.gov.br/pet. Acesso em: 20 abr. 2020.

CAPOVILLA, S.L.; SANTOS, A.A.A. (2001). Avaliação da influência de atividades extramuros no desenvolvimento pessoal de universitários. Psico-USF. Bragança Paulista, vol. 6, n. 2, pp.49-58.

FIOR, Camila Alves e MERCURI, Elizabeth. Formação universitária e flexibilidade curricular: importância das atividades obrigatórias e não obrigatórias. Psicol. educ. [online]. 2009, n.29, pp. 191-215. ISSN 1414-6975.

KUH, G. D. (1995). The other curriculum: out-of-class experiences associated with student learning and personal development. Journal of Higher Education. Ohio, vol. 66, n. 2, pp.123155.

NODARI, D. E.; LIMA, E. G. S.; MACIEL, C. E. O desempenho dos estudantes no vestibular e a permanência nos cursos de graduação da UNEMAT. Revista Avaliação do Ensino Superior, Campinas; Sorocaba, SP, v. 23, n. 02, p. 312-329, jul. 2018.

OLIVEIRA, Clarissa Tochetto de; SANTOS, Anelise Schaurich dos; DIAS, Ana Cristina Garcia. Percepções de Estudantes Universitários sobre a Realização de Atividades Extracurriculares na Graduação. Psicologia: Ciência e Profissão, [s.1.], v. 36, n. 4, p.864-876, dez. 2016. FapUNIFESP (SciELO). http://dx.doi.org/10.1590/1982-3703003052015.

PASCARELLA, E., e TERENZINI, P.T. (1991). How college affects students. São Francisco, Jossey-Bass.

. (2005). How college affects students: A third decade of research. Vol. 2. São

Francisco, Jossey-Bass.

RECKZIEGEL, Tatiana. O que muda com as novas diretrizes curriculares de 
Engenharia. 2019. Disponível em: https://desafiosdaeducacao.grupoa.com.br/novas-dens-deengenharia/. Acesso em: 20 abr. 2020.

SANTOS, Acácia Aparecida Angeli dos et al. Integração ao ensino superior e satisfação acadêmica em universitários. Psicologia: Ciência e Profissão, [s.1.], v. 33, n. 4, p.780-793, 2013. FapUNIFESP (SciELO). http://dx.doi.org/10.1590/s1414-98932013000400002.

SAVOY, Marcelo. Dicas CAD Arquitetônico. 2016. Disponível em:

http://cursosprime.com.br/teste/cad-arquitetura/dicas-cad-arquitetura/. Acesso em: 20 abr. 2020.

VALLE, A.et al. O potencial de transformação e melhoria da graduação exercido pelos grupos PET. Disponível em:

<http://www.abenge.org.br/sis_submetidos.php?acao=abrir\&evento=COBENGE18\&codigo= COBENGE18_00063_00001698.pdf>. Acesso em: 26 de abril de 2020.

VIEIRA, Alexandre. Novo treinamento de AutoCAD Civil 3D projeto viário. 2010.

Disponível em: https://infocads.wordpress.com/2010/08/23/novo-treinamento-de-autocadcivil-3d-projeto-viario/. Acesso em: 20 abr. 2020.

\title{
BEYOND THE TEACHING PLAN: EXTRACURRICULAR ACTIVITIES AND ITS IMPORTANCE
}

\begin{abstract}
The search for knowledge is an intrinsic human instinct and this goes far beyond starting a degree or a new language. There are several skills and competences of a professional that are needed today, in this way, the extracurricular activities are becoming a reality even more present in students of majorings. The main objective of this article is to emphasize the importance of participation in extra activities and personal and professional development. For this study, a survey was conducted with students participating in AutoCAD and Civil $3 D$ courses, taught by members of the Tutorial Education Program at the Federal University of Santa Catarina, Joinville campus.
\end{abstract}

Keywords: Extracurricular activities. Courses. Learning 